\title{
Cognitive load eliminates the global perceptual bias for unlimited exposure durations
}

\author{
Sarah Hoar • Karina J. Linnell
}

Published online: 15 January 2013

(C) Psychonomic Society, Inc. 2013

\begin{abstract}
In the present study, we used Navon-type Cognitive Psychology 9: 353-383 (1977) hierarchical patterns to demonstrate that cognitive load eliminates a global perceptual bias and enhances the representation of local elements at unlimited exposure durations. We added a cognitive-load manipulation to Kimchi and Palmer's Journal of Experimental Psychology: Human Perception and Performance 8:521-535 (1982) similarity-matching experiment with hierarchical patterns, and presented the stimuli for either unlimited or limited exposure durations. When exposures were unlimited, we demonstrated that observers exhibited a global bias under low, but not under high, cognitive load (Exp. 2). When exposures were limited, however, cognitive load exerted no effect, and the global bias remained (Exp. 1). We suggest that (1) these findings are best reconciled by proposing two stages in the representation of global structure, namely construction and maintenance; (2) the construction and maintenance stages are isolated, respectively, by limited-duration and unlimitedduration paradigms; and (3) cognitive processes play an integral role only in the maintenance stage. Given that real-world vision is not driven by a series of brief stimulus exposures, and is therefore likely to reflect maintenance processes, we argue that unlimited-exposure paradigms are more suitable for addressing real-world perceptual biases. When unlimitedexposure paradigms are used, cognitive load eliminates the commonly reported global bias.
\end{abstract}

Keywords Navon stimuli · Cognitive load · Global-local · Perceptual bias

\footnotetext{
S. Hoar $(\bowtie) \cdot$ K. J. Linnell

Department of Psychology, Goldsmiths, University of London, London, UK

e-mail: s.hoar@gold.ac.uk
}

The visual world is inherently hierarchical (Navon, 1977; Palmer, 1975); to use a common analogy, the forest exists as a global configuration of individual trees. In the Western world, there is a well-cited "global precedence" (Navon, 1977; see Kimchi, 1992, for a review), in that the analysis of visual information is initially dominated by the processing of global structure. This has been explained in terms of how the brain processes spatial-frequency information; lower spatial frequencies, which carry more global-level information, are processed faster than the higher spatial frequencies, which define more local detail (e.g., Shulman, Sullivan, Gish, \& Sakoda, 1986). The processing precedence of global information has, however, been demonstrated when stimulus presentation time is restricted, and this would not necessarily translate into a global bias in situations in which stimulus presentation time is unrestricted (arguably more akin to real-world viewing conditions). In the present study, we argue that for the global form to be most "salient in the final percept" (Kimchi, 1992, p. 26), the initial dominance of global structure must be maintained by cognitive processes; imposing a load on cognitive resources weakens the structural cohesiveness of the global form and increases the relative strength of local information. As the world is full of cognitive distractions, this has fundamental implications for the way in which visual information is represented.

The issue of global precedence was first explored by Navon (1977) using "hierarchical patterns," in which many small letters (e.g., $\mathrm{S}$ or $\mathrm{H}$ ) were arranged into the shape of a large letter (e.g., an $\mathrm{S}$ or $\mathrm{H}$ ), reflecting distinct local and global levels, respectively. When these hierarchical stimuli were presented very briefly, reaction times were faster to the identity of the global level, and global-to-local interference was observed (with an absence of local-to-global interference), suggesting that the global structure could not be ignored. Despite evidence supporting global precedence when exposure times are limited, 
a phenomenon referred to as orthographic satiation in Chinese characters (Cheng \& Lan, 2011; Cheng \& Wu, 1994) and Gestaltzerfall of Japanese kanji characters (Ninose \& Gyoba, 1996, 2002) suggests that the global structure must be maintained. Ninose and Gyoba (1996) demonstrated that kanji characters could be recognised very quickly by experienced readers, but after prolonged viewing (in the region of $25 \mathrm{~s}$ ), an uncertainty about the orthographical correctness of the perceptual whole emerged. The authors termed this Gestaltzerfall and concluded that kanji were initially represented within the visual system as perceptual wholes but that their structural cohesiveness was disrupted by prolonged viewing. In a later study, Ninose and Gyoba (2003) replicated their results when using hierarchical patterns.

More recently, evidence has suggested that the maintenance of global structure may induce cognitive fatigue. Helton, Hayrynen, and Schaeffer (2009) demonstrated that prolonged time performing a global speeded-response task not only impaired responses to the global level of hierarchical patterns, but also resulted in elevated right tympanic membrane temperature (TMT), a physiological marker that increases as cortical activation decreases; sustained attention to the local level during a local speeded-response task, however, had neither a behavioral nor a physiological effect. This was taken to reflect an increase in cognitive fatigue as a result of sustained attention only to the global level.

The work of Helton et al. (2009) and the phenomenon of Gestaltzerfall (Ninose \& Gyoba, 2003) suggest that cognitive resources are necessary to maintain the global representation beyond its initial construction, consistent with the idea that visual representations change across time (termed microgenesis; see, e.g., Bachmann, 2000; Flavell \& Draguns, 1957; Kimchi, 1998; Werner, 1956). We use the term construction to denote the perceptual processes that underlie the automatic formation of a global percept, akin to Koffka's (1935) shapegiving forces, and that are underpinned by the temporal precedence of low-spatial-frequency information (e.g., Shulman et al., 1986; Sripati \& Olson, 2009). However, Gestaltzerfall suggests that the representation of global structure must be kept active, and it is this process that we refer to as maintenance.

We investigated the involvement of cognitive resources in the maintenance of a global percept by observing how performance of a secondary task designed to engage working memory or cognitive resources-termed cognitive load-affected the representation of hierarchical information. As we suggest that the maintenance, but not the construction, of global structure requires cognitive resources, we would not expect cognitive load to have any effect on a limited-exposure task in which perception is dominated by global structure. However, cognitive load would serve to disrupt the maintenance processes involved in keeping the global representation active on an unlimited-exposure task and would accelerate Gestaltzerfall. As real-world vision is not driven by a series of brief exposures, and arguably reflects maintenance processes, we suggest that unlimited exposure durations - which allow observers to look at stimuli for as long as they like - are more suitable for investigating the effect of cognitive load on the representation of hierarchical information in real-world scenarios.

Although speeded-response paradigms are most commonly used with Navon (1977) hierarchical patterns, Navon (2003) suggested that similarity-matching paradigms (e.g., Kimchi \& Palmer, 1982) — in which participants match hierarchical patterns according to perceived similarity at either the local or the global level - are better suited to addressing which level of a hierarchical pattern is "given more weight in highorder processes" (p. 275). If cognitive load does affect the maintenance of the global percept and causes it to decay at an accelerated rate, we would expect fewer global matches to be made under high cognitive load when the exposure duration is unlimited (Exp. 2), but not when it is limited (Exp. 1).

\section{Experiment 1}

To investigate the effect of cognitive load on the construction of the global percept, we added a cognitive-load manipulation to Kimchi and Palmer's (1982) similarity-matching task and limited the exposure duration of the patterns; as limited-exposure tasks generally reveal a global bias (see Kimchi, 1992), we expected to illustrate this here and predicted that cognitive load would exert no effect.

The proportion of global matches was taken to reflect the relative strength of the representation of the global structure; if this proportion exceeded .5 , we interpreted this as a global bias. Kimchi and Palmer (1982) demonstrated that the proportion of global matches increases as the relative density of the individual elements increases; by including a patterndensity manipulation in the present study, we could observe whether cognitive load affected all densities equally.

\section{Method}

Design A mixed design was used, with pattern density (low, medium, and high) and display time (30 and $150 \mathrm{~ms})$ as the within-subjects variables, and cognitive load (low or high) as the between-subjects variable. Proportion of global matches was the dependent variable.

Participants A group of 40 individuals ( $M=24.98$ years; 28 female, 12 male) participated in the study and were reimbursed $£ 5$ for their time. All participants reported having normal or corrected-to-normal vision. The participants were alternately assigned to either the low- or the high-cognitive-load condition.

Apparatus and stimuli The experimental stimuli were presented on a Sony Trinitron CRT (F520) monitor using E- 
Prime version 1.2 (Psychology Software Tools Inc., Sharpsburg, PA). Patterns were presented on a white background and were small black triangles or squares configured to form large triangles or squares; the large shape could be either congruent (e.g., a large square formed of small squares) or incongruent (e.g., a large square formed of small triangles; see Fig. 1) with the small shapes. Each pattern subtended approximately $2.4^{\circ}$ of visual angle. The small squares subtended $1.2^{\circ}, 0.8^{\circ}$, and $0.6^{\circ}$, and the small triangles $1.0^{\circ}, 0.7^{\circ}$, and $0.5^{\circ}$ for the low, medium, and high densities, respectively. Each pattern was presented once at the top of a stimulus triad (see Fig. 1), with two comparison patterns below it, one of which it matched at the local level only, and the other of which it matched at the global level only. Each triad existed in two forms, with the locations of the comparison patterns switched. A total of 24 unique stimulus triads were used. Each stimulus triad was presented once for $30 \mathrm{~ms}$ and once for $150 \mathrm{~ms}$; the whole experiment consisted of 48 trials presented in a randomized order.

For the between-subjects cognitive-load manipulation, either one digit (low cognitive load) or six digits (high cognitive load) between 1 and 9 were pseudorandomly selected; for the high-cognitive-load condition, the digits were drawn without replacement.

Procedure The participants were seated $60 \mathrm{~cm}$ from a computer screen (maintained using a chinrest) and were informed that the task was to indicate, with a keypress, whether the pattern on the bottom left or right of the triad "looked most like" the pattern at the top, whilst remembering

\section{Â
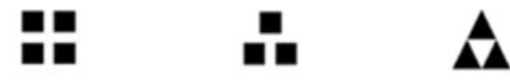 \\ H A
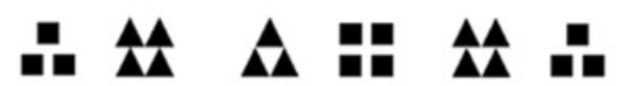
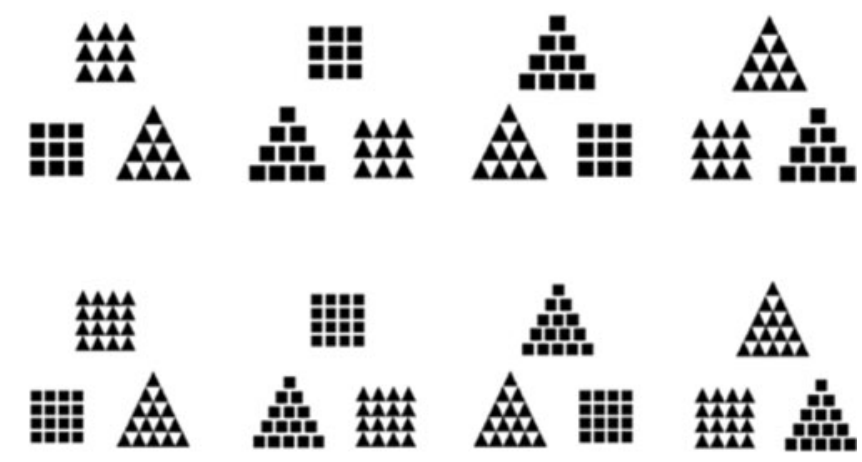

Fig. 1 An illustration of 12 of the 24 stimulus triads used. The remaining 12 trials were formed by switching the locations of the comparison patterns left to right. The top row depicts the low-density patterns, the middle row the medium-density patterns, and the bottom row the highdensity patterns. For the purpose of brevity, we omitted Kimchi and Palmer's (1982) highest-density patterns from our stimulus set either one (low cognitive load) or six (high cognitive load) digits. The digit(s) were presented (simultaneously) for $3,000 \mathrm{~ms}$, followed by a blank screen for $1,000 \mathrm{~ms}$, at the beginning of blocks of 24 trials; each trial began with a 1,000$\mathrm{ms}$ fixation cross before the stimulus triad was presented, centered on fixation, for either 30 or $150 \mathrm{~ms}$. After each block of 24 trials, participants were asked to type in the digit(s) that they had been remembering; for the high-cognitive-load manipulation, the digits were to be recalled in serial order, and participants were considered to have adequately recalled the digit string if at least five digits were recalled in the correct order. The procedure was then repeated for the remaining 24 trials.

\section{Results}

The cognitive-load task was performed adequately by all participants. A preliminary analysis of the matching data indicated that display time ( 30 vs. $150 \mathrm{~ms}$ ) had no effect on the responses $(p>.1)$; hence, this variable was excluded from further analysis. The proportions of global matches on the matching task were analyzed in a two-way mixed analysis of variance (ANOVA) with pattern density and cognitive load as the independent variables. A Bonferroni adjustment for multiple comparisons was applied to all post-hoc tests. We found no main effect of high $(M=.64, S E=.05)$ versus low $(M=.63$, $S E=.05)$ cognitive load on the proportions of global matches $(p>.1)$. Under both conditions of load, a global bias emerged: Two one-tailed $t$ tests demonstrated that the proportions of global matches differed significantly from .5 in both the lowcognitive-load $[t(19)=2.57, p<.05]$ and high-cognitive-load $[t(19)=2.36, p<.05]$ conditions.

A significant main effect of pattern density $[F(2,76)=$ 4.11, $p<.01, \eta=.7]$ demonstrated that more global matches were made to high-density $(M=.76, S E=.04)$ than to medium-density $(M=.69, S E=.04)$ patterns $[t$ (39) $=-3.46, p<.01]$, and that medium-density patterns in turn were matched more often on the global level than were low-density patterns $(M=.45, S E=.04)[t(39)=-$ $9.20, p<.01]$. This replicates the pattern observed by Kimchi and Palmer (1982) in their original task. Pattern density did not interact with cognitive load $(p>.1$; see Fig. 2); importantly, this illustrates that cognitive load did not affect the relationship between the density of the local elements and the likelihood that they would be matched according to their global structure.

\section{Discussion}

Our data show that, when exposure durations were limited, a global bias was exhibited under both low and high cognitive load. We concluded that cognitive load does not affect the construction of the global percept. 


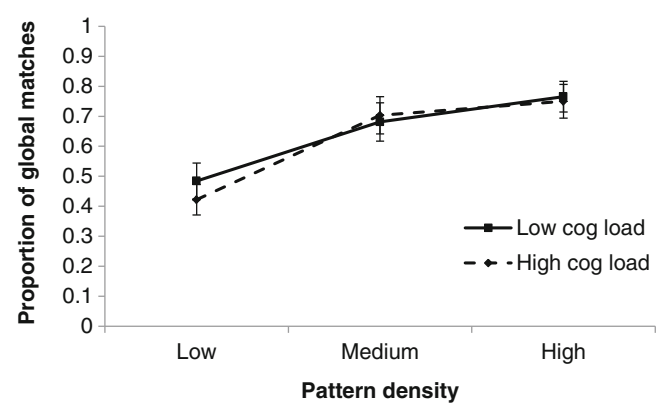

Fig. 2 Experiment 1: Limited exposure duration-Proportions of global matches made to hierarchical-patterns as a function of pattern density under both low and high cognitive load (error bars represent standard errors of the means)

\section{Experiment 2}

In Experiment 2, however, we investigated the role of cognitive resources in the maintenance of the global form. In this experiment, we allowed participants to view the stimuli for as long as they liked, by using unlimited exposure durations; as we have suggested that cognitive load affects the maintenance of the global form, we expected high cognitive load to eliminate the global bias observed in Experiment 1.

\section{Method}

Design All variables remained the same as in Experiment 1. Additionally, we recorded reaction times (RTs) to address whether any observed effects were due to the length of the viewing time.

Participants A group of 40 new individuals $(M=25.05$ years; 31 female, nine male) participated in the experiment and were reimbursed with $£ 5$. All of the participants reported having normal or corrected-to-normal vision. Participants were alternately assigned to either the low- or the high-cognitive-load condition.

Apparatus and stimuli The stimuli and apparatus were identical to those of Experiment 1.

Procedure The procedure was identical to that of Experiment 1 , with the exception that each stimulus triad was presented only once, for an unlimited duration. Now, only 24 trials were presented in one block. As with Experiment 1, participants in the high-cognitive-load condition were considered to have adequately recalled a digit string if at least five of the digits were recalled in the correct order.

\section{Results}

The cognitive-load task was adequately performed by all participants. The proportions of global matches on the matching task were analyzed with a two-way mixed ANOVA with pattern density and cognitive load as the independent variables. A Bonferroni adjustment for multiple comparisons was applied to all post-hoc tests. A main effect of cognitive load $[F(1,38)=5.49, p<.05, \eta=.13]$ indicated that participants under high cognitive load made significantly fewer global matches $(M=.41, S E=.07)$ than did those under low cognitive load $(M=.62, S E=.06)$. Cognitive load did not interact with pattern density (see Fig. 3). Two one-tailed $t$ tests demonstrated that, under low cognitive load, significantly more than $50 \%$ of the matches were made according to the global structure $[t(19)=2.16, p<$ $.05]$, whereas, under high cognitive load, less than $50 \%$ of the matches were global, although the latter effect was not significant $(p>.1)$.

A significant main effect of pattern density $[F(2,76)=$ 8.85, $p<.01, \eta=.19$; see Fig. 3] demonstrated that significantly more global matches were made to the high $(M=$ $.58, S E=.05)[t(39)=-3.46, p<.05]$ and medium $(M=.55$, $S E=.05)[t(39)=-3.49, p<.05]$ densities than to the low $(M=.46, S E=.04)$ density. As with Experiment 1, the absence of an interaction between pattern density and cognitive load suggests that cognitive load did not affect the relationship between the density of a pattern and the relative likelihood that it would be represented according to its global structure.

It was important to establish that the effect of load on matching was not driven by differences in inspection times. We repeated the two-way mixed ANOVA using RTs as the dependent variable and found that RTs under low cognitive load $(M=$ $1,352 \mathrm{~ms}, S E=158.6)$ were statistically identical to those under high cognitive load $(M=1,366.01 \mathrm{~ms}, S E=192.14)$; additionally, RTs did not interact with pattern density $(p>.1)$.

\section{Discussion}

Experiment 2 illustrated that the global bias demonstrated in Experiment 1 remained for participants under low cognitive load when exposure duration was unlimited. However, this

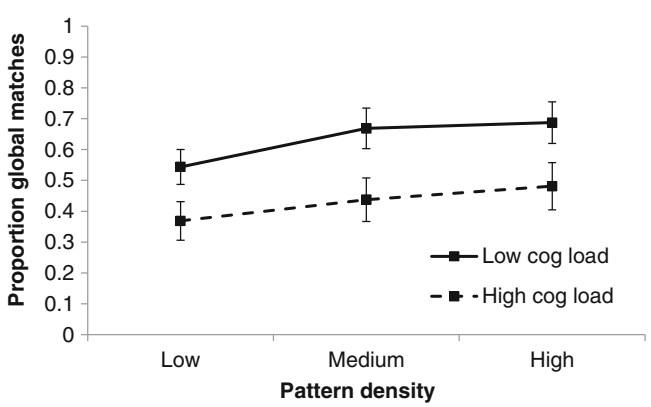

Fig. 3 Experiment 2: Unlimited exposure duration-Proportions of global matches made to hierarchical patterns as a function of pattern density under both low and high cognitive load (error bars represent standard errors of the means) 
global bias was eliminated when the task was performed under high cognitive load. We suggest that cognitive processes are involved in the maintenance, but not the construction, of the global form.

\section{General discussion}

In the present study, we tested the assumption that cognitive processes are required to maintain a global representation. We used a matching task that directly probed whether observers represent hierarchical patterns in terms of local elements or the global structure. We report that participants exhibited a global bias - regardless of cognitive loadwhen exposure durations were limited, whereas, when exposure durations were unlimited, high cognitive load was associated with a Gestaltzerfall of the global structure and a shift toward the representation of local elements. Our data suggest that cognitive load had no effect on the initial construction of the global percept but did weaken its stability, positioning cognitive resources as "shape-maintaining forces" (extending the terminology of Koffka, 1935).

One way of describing these findings would be in terms of the mechanism activation hypothesis (Lamb, London, Pond, \& Whitt, 1998; Lamb \& Yund, 1996). According to this hypothesis, identification of either the local or global level of a hierarchical pattern activates the relevant levelspecific neural mechanism. The early availability of global information activates the global-level mechanism at limited exposure durations under both low and high cognitive load. Maintaining the activation of the global-level mechanism, however, depends on the availability of cognitive resources, consistent with findings that right-hemisphere fatigue is induced both by performance of a cognitively demanding task (Lim et al., 2010) and by maintaining attention to global (but not to local) structure (Helton et al., 2009).

We suggest that our observed effect of cognitive load may translate into a change in the way in which real-world stimuli are represented; in essence, cognitive load could make it difficult to "see the wood for the trees." Humans are subjected to varying amounts of cognitive load. Whilst differences in load could be transitory, they could also be prolonged and translate into a chronic local bias; for example, powerless individuals have been shown to exhibit a more detailed processing style than the powerful (Guinote, 2007; Smith \& Trope, 2006) and powerlessness is a state which is associated with impaired executive functioning (Smith, Jostmann, Galinsky, \& van Dijk, 2008). Thus, any state which imposes a cognitive load may be associated with a more detailed processing style and may fundamentally alter how individuals perceive their environment.

Differences in processing style may also result from differences in situational factors, such as task difficulty, which impose differences in cognitive load. For example, the task of learning to read is cognitively demanding and as such may disrupt the structural cohesiveness of words which is fundamental to reading. Additionally, individuals with dyslexia or those who are less skilled readers may experience a greater cognitive load which may negatively affect the representation of words as perceptual wholes and hinder the task of reading further. We align our effect of cognitive load with the Gestaltzerfall of kanji characters observed by Ninose and Gyoba (1996); our data suggest that cognitive load may disrupt the organisational Gestalt of Kanji characters and make them more difficult to read.

In conclusion, we have illustrated the importance of using unlimited exposure durations when drawing inferences about real-world vision and perceptual biases. We have demonstrated that cognitive load eliminates the commonlyreported global bias and enhances the relative salience of local elements; we suggest that cognitive load affects the maintenance of a global percept but does not affect its initial construction, positioning cognitive processes as shapemaintaining forces.

Author note We thank Yaffa Yeshurun and two anonymous reviewers for their insightful comments on an earlier version of the manuscript.

\section{References}

Bachmann, T. (2000). Microgenetic approach to the conscious mind. Philadelphia: Benjamins.

Cheng, C. M., \& Lan, Y.-H. (2011). An implicit test of Chinese orthographic satiation. Reading and Writing, 24, 55-90.

Cheng, C. M., \& Wu, S. J. (1994). Orthographic satiation and disorganization in Chinese. Advances in the Study of Chinese Language Processing, 1, 1-30.

Flavell, J. H., \& Draguns, J. (1957). A microgenetic approach to perception and thought. Psychological Bulletin, 54, 197-217.

Guinote, A. (2007). Power affects basic cognition: Increased attentional inhibition and flexibility. Journal of Experimental Social Psychology, 43, 685-697.

Helton, W. S., Hayrynen, L., \& Schaeffer, D. (2009). Sustained attention to local and global target features is different: Performance and tympanic membrane temperature. Brain and Cognition, 71, 9-13.

Kimchi, R. (1992). Primacy of wholistic processing and global/local paradigm: A critical review. Psychological Bulletin, 112, 24-38. doi:10.1037/0033-2909.112.1.24

Kimchi, R. (1998). Uniform connectedness and grouping in the perceptual organization of hierarchical patterns. Journal of Experimental Psychology: Human Perception and Performance, 24, 1105-1118.

Kimchi, R., \& Palmer, S. E. (1982). Form and texture in hierarchically constructed patterns. Journal of Experimental Psychology. Human Perception and Performance, 8, 521-535. doi:10.1037/ 0096-1523.8.4.521

Koffka, K. (1935). Principles of Gestalt psychology. London: Lund Humphries.

Lamb, M. R., London, B., Pond, H. M., \& Whitt, K. A. (1998). Automatic and controlled processes in the analysis of hierarchical structure. Psychological Science, 9, 14-19. 
Lamb, M. R., \& Yund, E. W. (1996). Spatial frequency and attention: Effects of level-, target-, and location-repetition on the processing of global and local forms. Perception \& Psychophysics, 58, 363373.

Lim, J., Wu, W., Wang, J., Detre, J. A., Dinges, D. F., \& Rao, H. (2010). Imaging brain fatigue from sustained mental workload: An ASL perfusion study of the time-on-task effect. NeuroImage, 49, 3426-3435.

Navon, D. (1977). Forest before trees: The precedence of global features in visual perception. Cognitive Psychology, 9, 353-383. doi:10.1016/0010-0285(77)90012-3

Navon, D. (2003). What does a compound letter tell a psychologist's mind? Acta Psychologica, 114, 273-309.

Ninose, Y., \& Gyoba, J. (1996). Delays produced by prolonged viewing in the recognition of Kanji characters: Analysis of the "Gestaltzerfall" phenomenon. Japanese Journal of Psychology, 67, 227-231.

Ninose, Y., \& Gyoba, J. (2002). Analyses of the responsible factors for the delay effect produced by prolonged viewing in the recognizing of Kanji characters. Japanese Journal of Psychology, 73, 264 269.
Ninose, Y., \& Gyoba, J. (2003). The effect of prolonged viewing on the recognition of global and local levels of hierarchically constructed patterns. Acta Psychologica, 112, 233-242.

Palmer, S. E. (1975). Visual perception and world knowledge: Notes on a model of sensory-cognitive interaction. In D. A. Normam \& D. E. Rumelhart (Eds.), Explorations in cognition (pp. 279-307). San Francisco: Freeman.

Shulman, G. L., Sullivan, M. A., Gish, K., \& Sakoda, W. J. (1986). The role of spatial-frequency channels in the perception of local and global structure. Perception, 15, 259-273.

Smith, P. K., Jostmann, N. B., Galinsky, A. D., \& van Dijk, W. W. (2008). Lacking power impairs executive functions. Psychological Science, 19, 441-447.

Smith, P. K., \& Trope, Y. (2006). You focus on the forest when you're in charge of the trees: Power priming and abstract information processing. Journal of Personality and Social Psychology, 90, 578-596.

Sripati, A. P., \& Olson, C. R. (2009). Representing the forest before the trees: A global advantage effect in monkey inferotemporal cortex. Journal of Neuroscience, 29, 7788-7796.

Werner, H. (1956). Microgenesis and aphasia. Journal of Abnormal and Social Psychology, 52, 347-353. 